\title{
Lateral strength of nailed timber connections with decay
}

\author{
Kei Sawata ${ }^{1}\left[{ }^{\mathbb{D}} \cdot\right.$ Yoshihisa Sasaki $^{1}$
}

Received: 26 February 2018 / Accepted: 7 May 2018 / Published online: 6 June 2018

(c) The Japan Wood Research Society 2018

\begin{abstract}
Loading tests were conducted on nailed connections with decay due to a brown rot fungus. The effect of the decay on the lateral strength of nailed connections was investigated. After loading tests, the sound and decayed regions of a nailed connection were observed in the cross section, which was cut parallel to the grain through the nailed point. The nailed connections with decay showed a low load during initial deformation when the main and side members had a decayed region in the boundary between them. The nailed connections showed low load after yielding when the sound region in the main member decreased. The yield load of nailed connections with decay was calculated based on the yield theory. The model of calculations had sound and decay regions within a member. The yield load of nailed connections obtained by the calculation based on the yield theory agreed with the results obtained by experiments when significant decay in a direction parallel to the grain was observed in the main and side members. This result indicates that the yield theory can estimate the yield load of nailed connections not only with a sound member, but also a member that is partly or wholly decayed.
\end{abstract}

Keywords Yield theory $\cdot$ Yield mode $\cdot$ Brown rot fungus $\cdot$ Member thickness $\cdot$ Yield load

\section{Introduction}

Safety and serviceability are necessary functions of a structure, and those functions are often dependent on the stiffness and strength of the connections. The properties of the connections are required to persevere during its service period. Structural materials generally degrade as the service life increases. Wood is a material that is minimally affected by deterioration with age; however, it is affected by changes in humidity and may undergo biological deterioration under high humidity. One form of biological deterioration is decay. Decay causes a significant loss in the strength of the wood. The progress of decay differs with the direction of the grain and mycelium grows well along the longitudinal grain. Additionally, mycelial growth is not necessarily uniform within a wood. As a result, partial decay may happen in wood. When connections are decayed throughout, its properties would significantly degrade. However, for safety and serviceability of timber structures, it would be important to understand the properties of timber connections with part decay.

Kei Sawata

ksawata@for.agr.hokudai.ac.jp

1 Research Faculty of Agriculture, Hokkaido University, N9 W9, Sapporo 060-8589, Japan
There has been a great deal of research on the strengths of wood exposed to a wood-decaying fungus [1]. The effects of decay on wood strength have been investigated for compressive strength $[2,3]$, tensile strength [4], bending strength $[2,5,6]$, shear strength [2, 7], and hardness [2]. Although few studies have been conducted on timber connections with decay, reports have recently been increasing. Studies on timber connections with decay have been reported for dowelled connections [8] and nailed connections subjected to a lateral force. There have been several researches on the strengths of nailed connections exposed to a wood-decaying fungus. Studies on nailed connections have been conducted with side members composed of oriented strand board [9, 10], steel plate [11, 12], plywood, and medium density fiberboard [13]. These studies have indicated the decrease in strength of nailed connections caused by the decay.

Main and side members of nailed connections are composed of not only various materials, but also various dimensions. The connection geometry has effects on a yield mode, which depends on a distribution of embedding stress in members and a yield bending moment of nail. Further, the nail length in the main member affects a withdrawal resistance in the main member. Consequently, the shape of the load-slip curves of nailed connections, subjected to a lateral force, depends on the connection geometry [14]. There 
is little research that has examined the effects of decay on the strength of nailed connections with varying connection geometry. When decay is produced throughout main and side members, the strength of nailed connections will decrease because the embedding strength of wood [15] and withdrawal resistance in the main member [16] significantly decreases by decay. However, decay will not necessarily progress in a uniform manner within wood. Therefore, this study, conducted on the lateral loading tests of the decaytreated nailed connections with varying thicknesses of main and side members, investigated the effects of part decay in wood on the strength of nailed connections.

The strength of nailed connections with sound wood is often estimated by the calculation based on yield theory. The yield theory was developed by Johansen [17] and several standards have adopted it for the design of dowel-type timber connections subjected to lateral force $[18,19]$. The estimation based on yield theory has been conducted on not only the single and double shear connections with fastener placed with its axis perpendicular to the surface of the member but also the connections with inclined screw [20], nailed connections with interlayer [21], connections with multiple slotted-in steel plates [22], and connections in solid wood panels with cross layers [23]. The yield theory has been applied to various connections subjected to lateral force. If the strength of nailed connections with part decay could be estimated by yield theory, it would be useful for the evaluation of safety of structures. Therefore, to estimate the strength of nailed connections with part decay, the nailed connections with members that had sound and decayed regions were modeled based on the yield theory and its suitability was investigated. The embedding tests were conducted on sound and decaytreated wood to obtain the embedding strength, which was used in the calculation.

\section{Theory}

The calculation of the strength of dowel-type connections subjected to lateral force is based on a theory originally proposed by Johansen [17]. In this theory, the embedding of wood and bending of dowel are assumed to have perfect rigid plastic behavior, and several yield modes of the doweltype connections are assumed. The yield mode is based on the hypothesis that the dowel remains straight during yielding, and the yield moment of the dowel is reached at several points. The strength of the dowel-type connections differs according to yield mode. This theory is also applied to the nailed connections [19, 24].

When the regions with different embedding strength were within a member, the dowel-type connections showed a greater number of yield modes than in the case with uniform embedding strength [25]. The reason is that the yield mode varies according to the position of the plastic hinge and the rotation center of the dowel that rotate in the member. This study divided a member into two regions, which showed sound and decay status, because of modeling of nailed connections with part decay. In this case, main and side members each had five yield patterns as shown in Fig. 1. The yield mode of single shear nailed connections with part decay was decided by the combination of those patterns. Although the single shear nailed connections are well known to have six yield modes as shown in Fig. 2, when the main and side members were divided into two regions, the yield mode as shown in Fig. 2 was subdivided and the connections had 18 yield modes as shown in Table 1.

The strength estimation equations of the nailed connections were derived from the equilibrium equations of forces and moments based on yield theory. The equations corresponding to yield modes that prevent the rotation of nails are expressed as follows:

$P_{\mathrm{Ia}}=\left(f_{\mathrm{e} 11} \cdot t_{11}+f_{\mathrm{e} 12} \cdot t_{12}\right) \cdot d$,

$P_{\mathrm{Ib}}=\left(f_{\mathrm{e} 21} \cdot t_{21}+f_{\mathrm{e} 22} \cdot t_{22}\right) \cdot d$,

where $P_{\mathrm{Ia}}$ and $P_{\mathrm{Ib}}$ are the values $(\mathrm{N})$ corresponding to the mode Ia and Ib, respectively, $f_{\mathrm{e} i j}(i=1,2$ and $j=1,2)$ is the embedding strength $\left(\mathrm{N} / \mathrm{mm}^{2}\right)$ of the wood, $t_{i j}(i=1,2$ and $j=1,2)$ is the distance (mm) as shown in Fig. $3, d$ is the nail diameter $(\mathrm{mm})$.

When the nail is rotated in main and side members and the yield hinges are formed on nail, the moment of end of nail (Fig. 1) is

$M_{1}=\frac{1}{4}\left(A_{1} \cdot P^{2}+B_{1} \cdot P+C_{1}\right)$,

$M_{2}=\frac{1}{4}\left(A_{2} \cdot P^{2}+B_{2} \cdot P+C_{2}\right)$,

where $M_{1}$ and $M_{2}$ are moment, as shown in Fig. 1 , and $A_{k}$, $B_{k}$, and $C_{k}(k=1,2)$ are coefficients, as shown in Table 2.

By $M_{1}+M_{2}=0$ in the boundary between main and side members, the equations corresponding to yield modes as shown in Table 1 are obtained as follows:

$P=\frac{\sqrt{\left(B_{1}+B_{2}\right)^{2}-4\left(A_{1}+A_{2}\right)\left(C_{1}+C_{2}\right)}-\left(B_{1}+B_{2}\right)}{2\left(A_{1}+A_{2}\right)}$,

where $P$ is the values corresponding to each yield mode and $P=P_{\mathrm{II} n}, P_{\mathrm{IIIa} n}, P_{\mathrm{IIIb} n}$, or $P_{\mathrm{IV} n}(n=1-4)(\mathrm{N})$, as shown in Fig. 2.

Using the values of $P_{\mathrm{II} n}, P_{\mathrm{IIIIa} n}, P_{\mathrm{IIIb} n}$, or $P_{\mathrm{IV} n}$ which are the positive real number, the yield load of nailed connections $\left(P_{\mathrm{y}}\right)$ is obtained from following equation. 

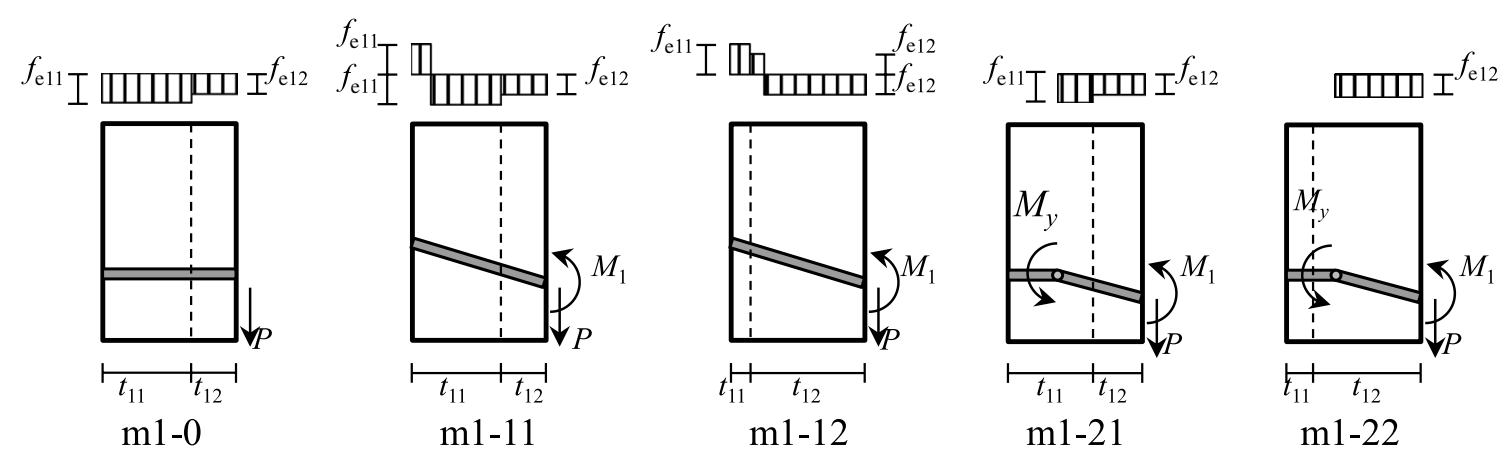

(a) Side member

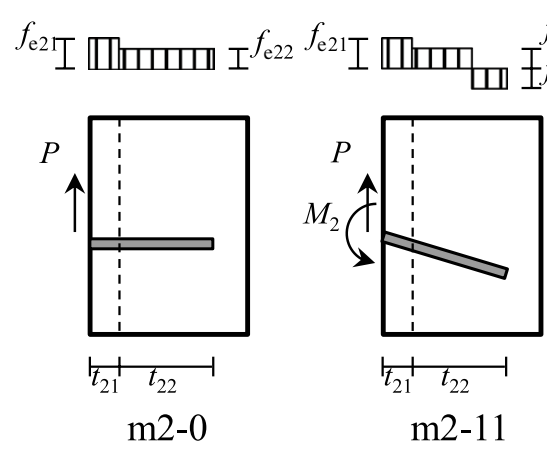

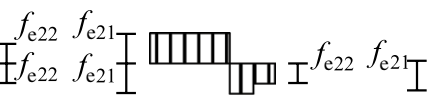
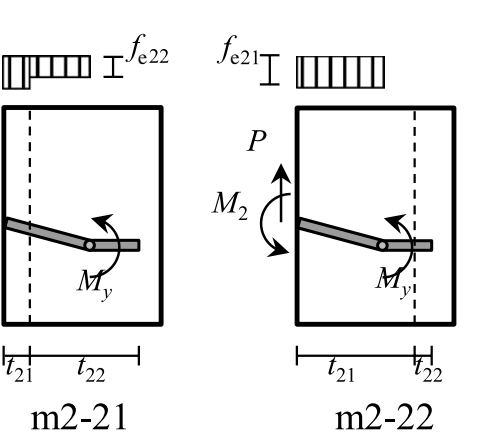

(b) Main member

Fig. 1 Yield pattern of main and side members. $f_{\mathrm{e} l l}, f_{\mathrm{e} l 2}, f_{\mathrm{e} 2 l}$, and $f_{\mathrm{e} 22}$ embedding strength corresponding to sound and decay status, $t_{11}$ and $t_{12}$ region with different status of side member, $t_{21}$ and $t_{22}$ region with different status of main member, $M_{1}$ and $M_{2}$ moment, $M_{y}$ yield moment, $P$ load
Fig. 2 Yield mode of nailed connection

$P_{\mathrm{y}}=\min \left\{\begin{array}{l}P_{\mathrm{Ia}} \\ P_{\mathrm{Ib}} \\ P_{\mathrm{II} n} \\ P_{\mathrm{III} n} \\ P_{\mathrm{IIIb} n} \\ P_{\mathrm{IV} n}\end{array} \quad(n=1-4)\right.$.

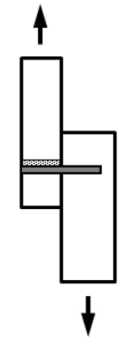

Mode Ia

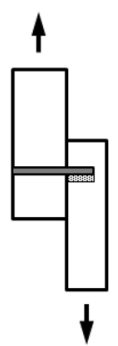

Mode Ib

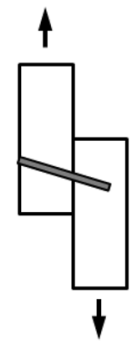

Mode II

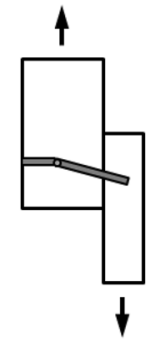

Mode IIIa

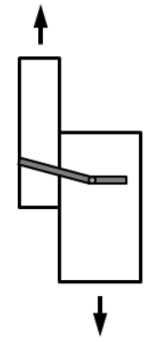

Mode IIIb

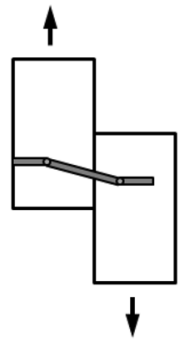

Mode IV

\section{Materials and methods}

\section{Nailed connection test}

The strength of the nailed connections was determined from tests using solid lumbers, Japanese fir (Abies sachalinensis), used for the main and side members. The main and side members were $250 \mathrm{~mm}$ long and $45 \mathrm{~mm}$ wide. The side member thickness and nail length in the main member were of three types, as shown in Table 3 . The distance from the point of the nail to the edge of the main member was $15 \mathrm{~mm}$. Control and decay-treated specimens 
Table 1 Yield mode

\begin{tabular}{llll}
\hline Mode & Value & \multicolumn{2}{l}{ Yield pattern } \\
\cline { 3 - 4 } & & Side member & Main member \\
\hline Ia & $P_{\text {Ia }}$ & $\mathrm{m} 1-0$ & - \\
Ib & $P_{\text {Ib }}$ & - & $\mathrm{m} 2-0$ \\
II1 & $P_{\text {III }}$ & $\mathrm{m} 1-11$ & $\mathrm{~m} 2-11$ \\
II2 & $P_{\text {III }}$ & $\mathrm{m} 1-11$ & $\mathrm{~m} 2-12$ \\
II3 & $P_{\text {II3 }}$ & $\mathrm{m} 1-12$ & $\mathrm{~m} 2-11$ \\
II4 & $P_{\text {III }}$ & $\mathrm{m} 1-12$ & $\mathrm{~m} 2-12$ \\
IIIa1 & $P_{\text {IIIa1 }}$ & $\mathrm{m} 1-21$ & $\mathrm{~m} 2-11$ \\
IIIa2 & $P_{\text {IIIa2 }}$ & $\mathrm{m} 1-21$ & $\mathrm{~m} 2-12$ \\
IIIa3 & $P_{\text {IIIa3 }}$ & $\mathrm{m} 1-22$ & $\mathrm{~m} 2-11$ \\
IIIa4 & $P_{\text {IIIa4 }}$ & $\mathrm{m} 1-22$ & $\mathrm{~m} 2-12$ \\
IIIb1 & $P_{\text {IIIb1 }}$ & $\mathrm{m} 1-11$ & $\mathrm{~m} 2-21$ \\
IIIb2 & $P_{\text {IIIb1 }}$ & $\mathrm{m} 1-11$ & $\mathrm{~m} 2-22$ \\
IIIb3 & $P_{\text {IIIb3 }}$ & $\mathrm{m} 1-12$ & $\mathrm{~m} 2-21$ \\
IIIb4 & $P_{\text {IIIb4 }}$ & $\mathrm{m} 1-12$ & $\mathrm{~m} 2-22$ \\
IV1 & $P_{\text {IV1 }}$ & $\mathrm{m} 1-21$ & $\mathrm{~m} 2-21$ \\
IV2 & $P_{\text {IV2 }}$ & $\mathrm{m} 1-21$ & $\mathrm{~m} 2-22$ \\
IV3 & $P_{\text {IV3 }}$ & $\mathrm{m} 1-22$ & $\mathrm{~m} 2-21$ \\
IV4 & $P_{\text {IV4 }}$ & $\mathrm{m} 1-22$ & $\mathrm{~m} 2-22$ \\
\hline
\end{tabular}

For the abbreviations of yield pattern, see Fig. 1

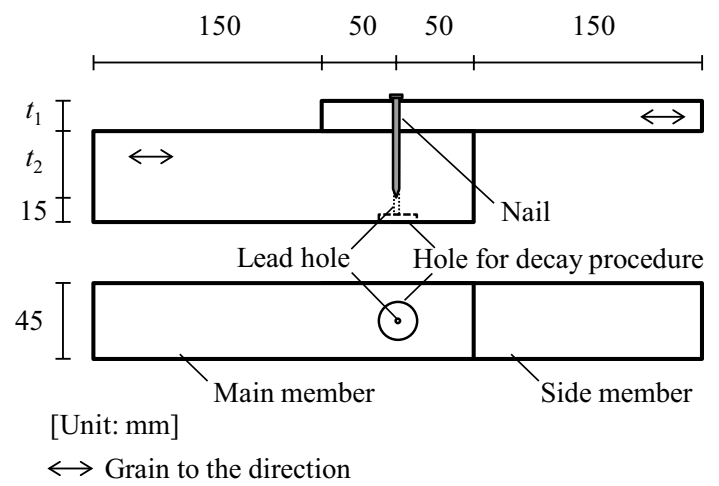

Fig. 3 Configuration of nailed connection specimen

were prepared from the end-matched group. The average wood density of an end-matched small specimen without defects was $381 \mathrm{~kg} / \mathrm{m}^{3}$ (standard deviation: $41.0 \mathrm{~kg} / \mathrm{m}^{3}$ ) at an average moisture content of $7.67 \%$. The main and side members had a nail lead hole of $3 \mathrm{~mm}$ diameter. The lead hole was drilled before the main and side members were immersed in a water bath, which will be described later. The main member was prepared with a hole of $25 \mathrm{~mm}$ diameter and $5 \mathrm{~mm}$ depth for the decay procedure, as shown in Fig. 3.

Prior to the decay procedure, the wood samples were immersed in a water bath for more than 2 weeks to increase their moisture content. The wood samples were placed in polyethylene bags with a filter and sterilized by heating to $121{ }^{\circ} \mathrm{C}$ for $15 \mathrm{~min}$. The hole for the decay procedure was filled with potato dextrose agar and a small block covered with mycelium was placed in the potato dextrose agar. A small block of Japanese fir was inoculated with a small piece of Fomitopsis palustris (Berk. et Curt.; a brown rot fungus) on a mycelium mat, and the nutrient solution consisted of tap water, including $4 \%$ D-glucose, $0.3 \%$ peptone, and $1.5 \%$ malt extract $[7,16]$. The main and side members were assembled with paper strings and the lead hole of the main member was aligned with that of the side member. The authors expected mycelial growth to begin along the lead hole. The inoculated wood samples were incubated at $25{ }^{\circ} \mathrm{C}$ and $83 \%$ relative humidity for 9 and 21 weeks.

When wood with decay is dried, the wood shows significantly shrinkage deformation. If the decay-treated specimens are dried after decay procedure, the specimens might not be set up on testing equipment because of the significant deformation. The strength of wood with moisture content above the fiber saturation point is usually, almost, constant and the same observation has been obtained from the report for the embedding strength of wood [15]. Therefore, to avoid the significant deformation and to increase moisture content up to above the fiber saturation point, the wood samples were immersed in a water bath for more than 1 week after the decay procedure. Control wood samples were also immersed in a water bath prior to the lateral loading tests. The moisture content of the control and inoculated wood samples at the time of the lateral loading tests were 70.3-166 and $78.1-279 \%$, respectively.

Mycelium grows well under conditions of moisture content above the fiber saturation point, and the exposure of nailed connections to high moisture encourages corrosion. When rust forms on the surface of the nail shank, the surface roughness of the shank increases, therefore, nail corrosion leads to an increase in strength of nailed connections. When the nail further corrodes, the nail shank decreases and the strength of nailed connections are decreased [26]. The strength of nailed connections exposed to fungal attack is affected by both wood decay and nail corrosion. In this study, the nails were driven into wood after the decay procedure because the effects of rust on the nail on the strength of nailed connections were excluded, and the effects of the decay were investigated.

The nails used in nailed connection tests were a CN65 nail having a nominal diameter of $3.33 \pm 0.10 \mathrm{~mm}$ and a nominal length of $63.5 \pm 1.6 \mathrm{~mm}[27,28]$. A nominal length is the length from nail point to nail head and the length of nail shank was $58 \mathrm{~mm}$. The average bending strength corresponding to an angle of $45^{\circ}$ of the $\mathrm{CN} 65$ nail was obtained from the bending tests according to ISO 10984-1 [29], and was $1379 \mathrm{~N} / \mathrm{mm}^{2}$. The yield bending strength of the nails, 
Table 2 Coefficients of quadric equation for moment

\begin{tabular}{llll}
\hline Yield pattern & $A_{1}$ & $B_{1}$ & $C_{1}$ \\
\hline $\mathrm{m} 1-11$ & $I_{11}$ & $2 d \cdot I_{11}\left(J_{11}-J_{12}+2 f_{\mathrm{e} 11} \cdot t_{12}\right)$ & $d^{2} \cdot I_{11}\left(J_{12}{ }^{2}-J_{11}{ }^{2}\right)-2 d \cdot J_{12}\left(t_{11}+t_{12}\right)$ \\
$\mathrm{m} 1-12$ & $I_{12}$ & $2 d \cdot I_{12}\left(J_{11}+J_{12}\right)$ & $d^{2} \cdot I_{12}\left(J_{11}{ }^{2}-J_{12}{ }^{2}\right)-2 d \cdot J_{11}\left(t_{11}+t_{12}\right)$ \\
$\mathrm{m} 1-21$ & $2 I_{11}$ & $4\left(t_{12}-d \cdot I_{11} \cdot J_{12}\right)$ & $2 d^{2} \cdot I_{11} \cdot J_{12}\left(J_{12}-f_{\mathrm{e} 11} \cdot t_{12}\right)-4 M_{\mathrm{y}}$ \\
$\mathrm{m} 1-22$ & $2 I_{12}$ & 0 & $-4 M_{\mathrm{y}}$ \\
\hline Yield pattern & $A_{2}$ & $B_{2}$ & $C_{2}$ \\
\hline $\mathrm{m} 2-11$ & $I_{22}$ & $2 d \cdot I_{22}\left(J_{22}-J_{21}+2 f_{\mathrm{e} 22} \cdot t_{21}\right)$ & $d^{2} \cdot I_{22}\left(J_{21}{ }^{2}-J_{22}{ }^{2}\right)-2 d \cdot J_{21}\left(t_{21}+t_{22}\right)$ \\
$\mathrm{m} 2-12$ & $I_{21}$ & $2 d \cdot I_{21}\left(J_{21}+J_{22}\right)$ & $d^{2} \cdot I_{21}\left(J_{22}{ }^{2}-J_{21}{ }^{2}\right)-2 d \cdot J_{22}\left(t_{21}+t_{22}\right)$ \\
$\mathrm{m} 2-21$ & $2 I_{22}$ & $4\left(t_{21}-d \cdot I_{22} \cdot J_{21}\right)$ & $2 d^{2} \cdot I_{22} \cdot J_{21}\left(J_{21}-f_{\mathrm{e} 22} \cdot t_{21}\right)-4 M_{y}$ \\
$\mathrm{~m} 2-22$ & $2 I_{21}$ & 0 & $-4 M_{\mathrm{y}}$ \\
\hline
\end{tabular}

$I_{i j}=1 /\left(f_{\mathrm{e} i j} \cdot d\right), J_{i j}=f_{\mathrm{e} i j} \cdot t_{i j}$
Table 3 Nailed connection tests specimen

\begin{tabular}{llllll}
\hline Type & \multicolumn{2}{l}{ Side member } & & \multicolumn{2}{l}{ Main member } \\
\cline { 2 - 3 } & $t_{1}(\mathrm{~mm})$ & $t_{1} / l_{n}$ & & $t_{2}(\mathrm{~mm})$ & $t_{2} / l_{n}$ \\
\hline S3M7 & 20 & 0.34 & & 38 & 0.66 \\
S6M4 & 33 & 0.57 & & 25 & 0.43 \\
S8M2 & 45 & 0.78 & & 13 & 0.22 \\
\hline
\end{tabular}

according to EN12512 [30], was $599 \mathrm{~N} / \mathrm{mm}^{2}$. The main and side members were connected with a nail after those were immersed in a water bath. The nails were hand-driven into the radial direction to the annual ring of the main and side members and the nailed connections were immediately tested after nailing. The nailed connections had three specifications that differed with the thickness of the members. Each specification consisted of 15 replicates. Five specimens were control specimens and ten specimens were decay-treated specimens.

The main and side members of a nailed connection were loaded parallel to the grain as shown in Fig. 4a. The relative slips between the main and side members of the nailed connection were measured by two displacement transducers. A load was applied up to a relative slip of $0.25 \mathrm{~mm}$ and, subsequently, was reduced to $0 \mathrm{~mm}$. This step was repeated three times. Then, a cyclic test was repeated three times to produce the relative slips of $0.5,0.75,1.0,2.0$, and $4 \mathrm{~mm}$. The slip level was determined from the load-slip curves obtained from the preliminary loading tests of the nailed connections. The loading procedure was adopted because the degradation of wood was investigated in the unloaded area of the nailed connection. The tests were terminated when the load decreased to $60 \%$ of the maximum load or when the relative slip reached $40 \mathrm{~mm}$.

\section{Embedding test}

The dimensions of the embedding test specimens, which were cut from Japanese fir solid lumber, were $95 \mathrm{~mm}$ long, $45 \mathrm{~mm}$ wide, and $12 \mathrm{~mm}$ thick. Control and decay-treated specimens were prepared from the end-matched group. The average wood density of a specimen before decay procedure was $387 \mathrm{~kg} / \mathrm{m}^{3}$ (standard deviation: $17.1 \mathrm{~kg} / \mathrm{m}^{3}$ ) at

Fig. 4 Setup of lateral loading tests for nailed connection and embedding tests

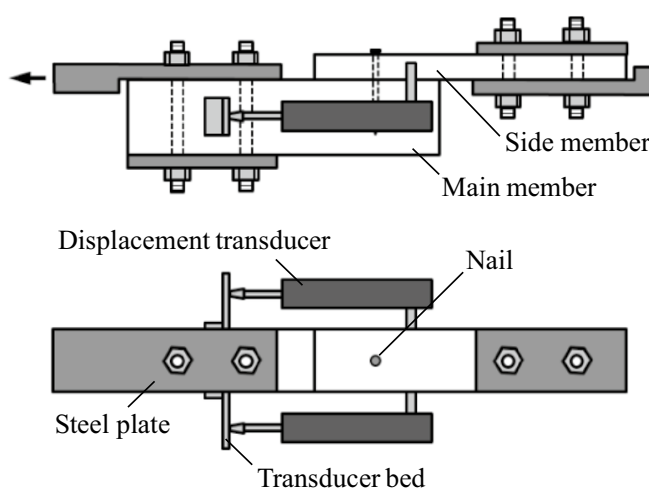

(a) Nailed connection test

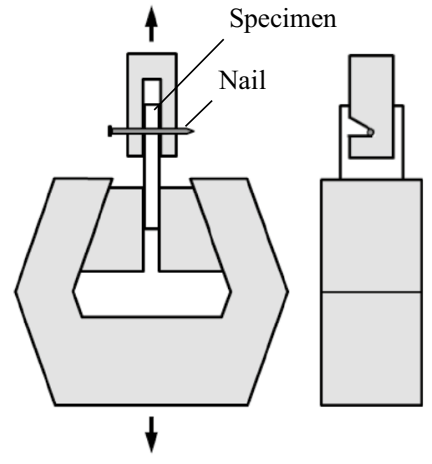

(b) Embedding test 
an average moisture content of $12.7 \%$. The nail used was a CN65 nail, a nail lead hole was $3 \mathrm{~mm}$ diameter, and the end distance was $20 \mathrm{~mm}$.

The specimens were sterilized and placed in a polypropylene container containing the fungus culture of Fomitopsis palustris. Nutrient solution was tap water containing $4 \%$ D-glucose, $0.3 \%$ peptone, and $1.5 \%$ malt extract. The incubation was performed at $25{ }^{\circ} \mathrm{C}$ and $83 \%$ relative humidity for 6,9 , and 12 weeks. The embedding test specimens were immersed in a water bath for more than 1 week prior to the loading tests to avoid significantly shrinkage deformation, as described above. The moisture content of the control and inoculated specimens at the time of the loading tests were $120-154$ and $178-273 \%$, respectively.

After the above procedure, CN65 nails were hand-driven into the radial direction to the annual ring of the specimens and the embedding tests were immediately carried out after nailing. The monotonic loading tests parallel to the grain were carried out, as shown in Fig. 4b. The tests were terminated when the load decreased to $60 \%$ of the maximum load or when the slip reached more than $3.5 \mathrm{~mm}$. The control specimens were 12 and the decay-treated specimens were 18 .

\section{Results and discussion}

\section{Embedding strength}

Figure 5 shows the representative embedding stress-displacement curves of the embedding specimens that had different degrees of decay. The mass loss was calculated from the weight of end-matched control and decay-treated specimens after embedding tests as follows:

$\mathrm{ML}=\frac{W_{\mathrm{CO}}-W_{\mathrm{DO}}}{W_{\mathrm{CO}}} \times 100$,

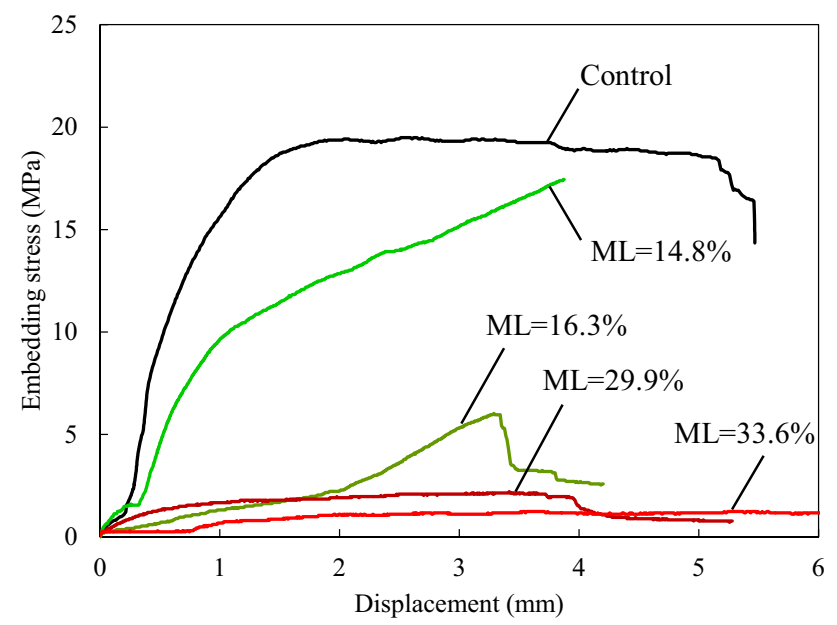

Fig. 5 Relationship between the embedding stress and displacement. $M L$ mass loss where $\mathrm{ML}$ is the mass loss (\%), $W_{\mathrm{CO}}$ is the weight of ovendry control specimen $(\mathrm{g}), W_{\mathrm{DO}}$ is the weight of oven-dry decay-treated specimen $(\mathrm{g})$.

The control specimen show almost constant load after displacement of $2 \mathrm{~mm}$. The decay-treated specimens with mass loss of 14.8 and $16.3 \%$ show a increase in the load up to the termination of test and that with mass loss of 29.9 and $33.6 \%$ show almost constant load after displacement of $2 \mathrm{~mm}$. The former had both of sound and decayed regions in the nailing point and the latter had only decayed region in the nailing point. In this study, to obtain the embedding strength of sound and decay wood, the embedding strength was defined as the maximum embedding stress up to $2 \mathrm{~mm}$ displacement.

Figure 6 shows the relations between the embedding strength and mass loss. When the mass loss was in the range of $8-20 \%$, the specimens showed a large variable of embedding strength. The specimens with mass loss of greater than $20 \%$ showed significantly lower embedding strength than the control specimens. The visual feature by decay was not clear during embedding test; however, a discoloration, shrinkage, and collapse were obviously observed by seasoning after embedding test. The specimens with mass loss of lower than $20 \%$ showed partly a discoloration, shrinkage, and collapse in the nailing point and all specimens with mass loss of greater than $20 \%$ showed those visual features by decay in the overall nailing point. Therefore, the embedding strength with mass loss of greater than $20 \%$ was defined as the embedding strength of decay region in this study. The embedding strength of sound region, which was obtained from the embedding tests of control specimens, was $18.3 \mathrm{~N} /$ $\mathrm{mm}^{2}$ and that of decay region was $1.26 \mathrm{~N} / \mathrm{mm}^{2}$.

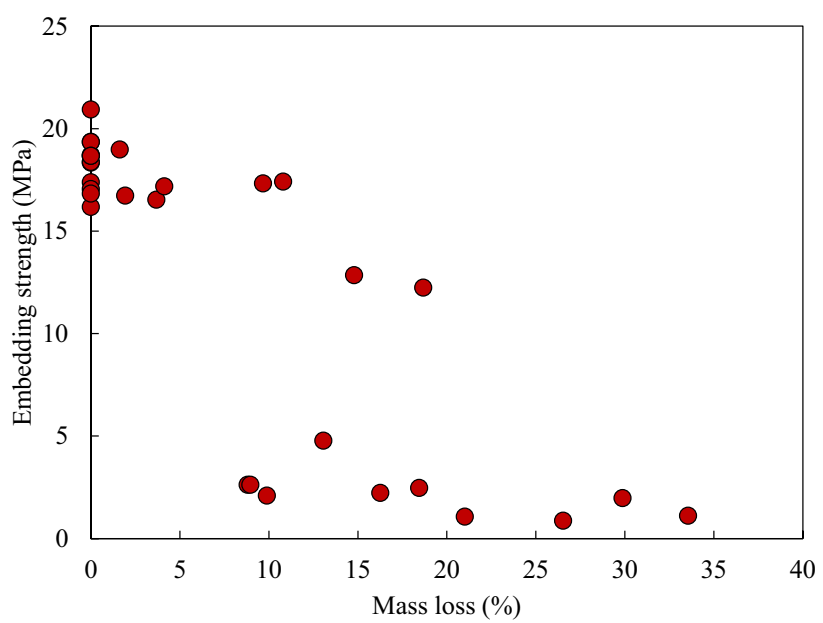

Fig. 6 Relationship between embedding strength and mass loss 
Fig. 7 Cross section of nailed connection after loading test. $[S]$ sound status, $[D]$ decay status

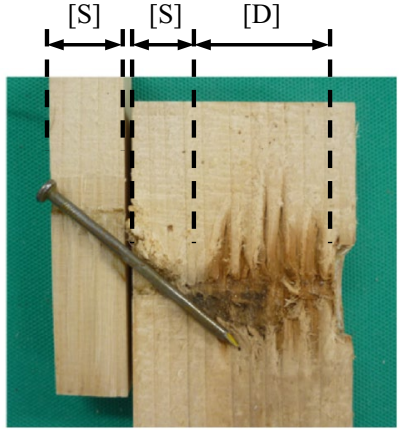

(a) S3M7-4 specimen

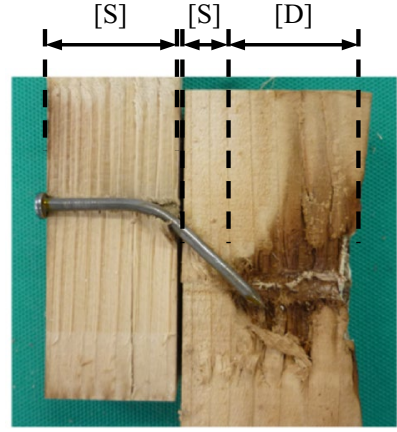

(b) S6M4-7 specimen

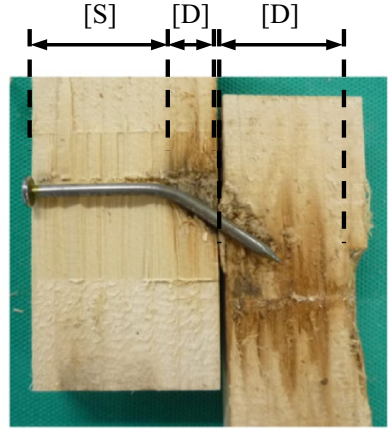

(c) S8M2-8 specimen

\section{Decay pattern of nailed connection}

After the loading tests, the nailed connections were cut parallel to the grain through the nailed point, and were conditioned to air-dry humidity. Figure 7 shows the typical cross section of nailed connections after the loading test. A discoloration, crack across to the grain, shrinkage, and collapse, which were caused by decay, were observed in main and side members by seasoning. Some specimens produced decay throughout wood members and others produced part decay in wood members. In the case of the specimens with the part decay, a member was mostly distinguishable in two regions, which were the sound and decayed regions. The sound and decayed regions parallel to the nail axis were measured to obtain the degree of decay of nailed connections. Table 4 shows the length of sound and decayed regions of individual specimens. Because the presence or absence of decay and the length of decayed region differed with the specimens, the specimens with various degrees of decay could be obtained.

\section{Load-slip curve of nailed connection}

Figure 8 shows the representative load-slip curves of the nailed connections that had different degrees of decay. The load of the S3M7 control specimen shows a linear increase up to the yield point and a continuous increase after yielding. The S3M7-2 specimen, which had a sound side member and a partly decayed main member, exhibits almost the same load-slip curve as the S3M7 control specimen. The S3M7-9 specimen, which had a sound side member and a main member with no sound region, shows a smooth increase in the load during the initial deformation and a slight increase after yielding. The S3M7-8 specimen had a side member with no sound region and a partly decayed main member. The S3M7-10 specimen had the side and main members with no sound region. These specimens show a low load at the initial deformation, with the load of the S3M7-10 specimen being smaller than that of the S3M7-8 specimen.

A sound nailed connection subjected to a lateral force will develop a mechanism as follows: An embedding
Table 4 Length of sound and decayed regions of decay-treated specimens

\begin{tabular}{|c|c|c|c|c|}
\hline \multirow[t]{2}{*}{ Specimens } & \multicolumn{2}{|c|}{ Side member } & \multicolumn{2}{|c|}{ Main member } \\
\hline & $t_{11}(\mathrm{~mm})$ & $t_{12}(\mathrm{~mm})$ & $t_{21}(\mathrm{~mm})$ & $t_{22}(\mathrm{~mm})$ \\
\hline S3M7-1 & - & $20[\mathrm{~S}]$ & $38[\mathrm{~S}]$ & - \\
\hline S3M7-2 & - & $20[S]$ & $25[\mathrm{~S}]$ & 13 [D] \\
\hline S3M7-3 & - & $20[\mathrm{~S}]$ & $24[\mathrm{~S}]$ & 14 [D] \\
\hline S3M7-4 & - & $20[\mathrm{~S}]$ & $19[\mathrm{~S}]$ & $19[\mathrm{D}]$ \\
\hline S3M7-5 & - & $20[\mathrm{~S}]$ & $15[\mathrm{~S}]$ & $23[\mathrm{D}]$ \\
\hline S3M7-6 & - & $20[S]$ & $12[\mathrm{~S}]$ & $26[\mathrm{D}]$ \\
\hline S3M7-7 & - & $20[S]$ & $11[\mathrm{~S}]$ & $27[\mathrm{D}]$ \\
\hline S3M7-8 & - & 20 [D] & $27[S]$ & 11 [D] \\
\hline S3M7-9 & - & $20[\mathrm{~S}]$ & 38 [D] & - \\
\hline S3M7-10 & - & $20[\mathrm{D}]$ & 38 [D] & - \\
\hline S6M4-1 & - & $33[\mathrm{~S}]$ & $25[\mathrm{~S}]$ & - \\
\hline S6M4-2 & - & $33[\mathrm{~S}]$ & $25[\mathrm{~S}]$ & - \\
\hline S6M4-3 & - & $33[\mathrm{~S}]$ & $25[\mathrm{~S}]$ & - \\
\hline S6M4-4 & - & $33[\mathrm{~S}]$ & $25[\mathrm{~S}]$ & - \\
\hline S6M4-5 & - & $33[\mathrm{~S}]$ & $14[\mathrm{~S}]$ & $11[\mathrm{D}]$ \\
\hline S6M4-6 & - & $33[\mathrm{~S}]$ & 11 [D] & $14[\mathrm{~S}]$ \\
\hline S6M4-7 & - & $33[\mathrm{~S}]$ & $12[\mathrm{~S}]$ & 13 [D] \\
\hline S6M4-8 & $27[\mathrm{~S}]$ & 6 [D] & 25 [D] & - \\
\hline S6M4-9 & 23 [D] & $10[\mathrm{~S}]$ & $12[\mathrm{~S}]$ & 13 [D] \\
\hline S6M4-10 & - & 33 [D] & $13[\mathrm{~S}]$ & 12 [D] \\
\hline S8M2-1 & - & $45[\mathrm{~S}]$ & $13[\mathrm{~S}]$ & - \\
\hline S8M2-2 & - & $45[\mathrm{~S}]$ & $8[\mathrm{~S}]$ & 5 [D] \\
\hline S8M2-3 & - & $45[\mathrm{~S}]$ & 13 [D] & - \\
\hline S8M2-4 & - & $45[\mathrm{~S}]$ & 13 [D] & - \\
\hline S8M2-5 & - & $45[\mathrm{~S}]$ & 13 [D] & - \\
\hline S8M2-6 & - & $45[\mathrm{~S}]$ & 13 [D] & - \\
\hline S8M2-7 & 23 [D] & $22[\mathrm{~S}]$ & $13[\mathrm{~S}]$ & - \\
\hline S8M2-8 & $34[\mathrm{~S}]$ & $11[\mathrm{D}]$ & 13 [D] & - \\
\hline S8M2-9 & - & $45[\mathrm{D}]$ & 13 [D] & - \\
\hline S8M2-10 & - & $45[\mathrm{D}]$ & 13 [D] & - \\
\hline
\end{tabular}

$t_{11}, t_{12}, t_{21}$, and $t_{22}$, see Fig. 1

$S$ sound status, $D$ decayed status 


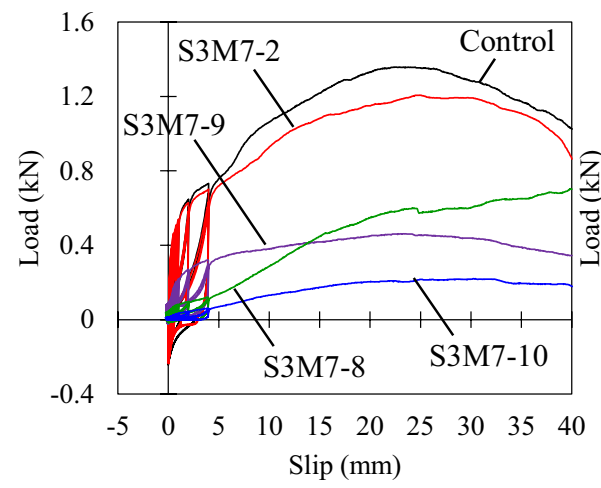

(a) S3M7 specimens

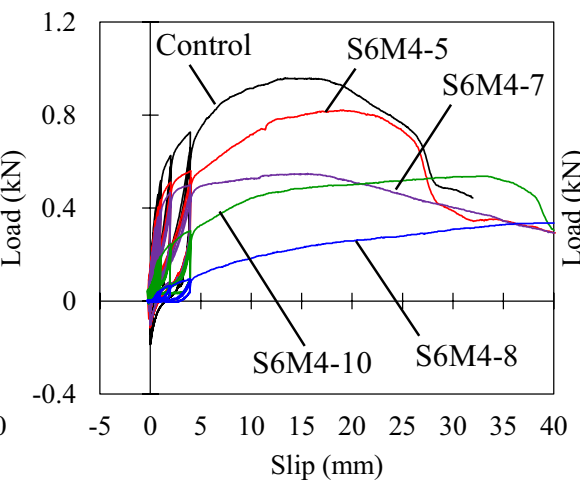

(b) S6M4 specimens

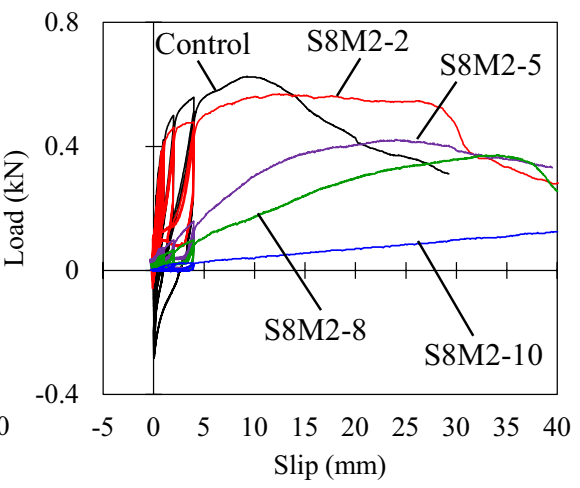

(c) S8M2 specimens

Fig. 8 Relationship between the load and slip of nailed connections

stress in the main and side members is concentrated on the boundary between those members during the initial deformation [31]. Thereafter, a plastic hinge is produced on the nail and a nailed connection reaches a yielding condition. A nailed connection shows an increase in the load by increasing both the withdrawal resistance in the main member and the nail-head pull-through resistance in the side member after yielding. When the nailed connections of the S3M7 group had sound regions in the boundary between the main and side members, the connections showed a sharp increase in the load during the initial deformation. The connections showed a low load during the initial deformation when there was a small sound region in the boundary between the main and side members, and showed a low load after yielding when the main member had no sound regions. This is because the decay of the main member causes a decrease in the embedding strength and withdrawal resistance [16]. Support for this observation can be understood by the mechanism described above.

The S6M4-5 and S6M4-7 specimens had sound regions in the boundary between the main and side members. These showed a sharp increase in the load during the initial deformation. However, the shape of the load-slip curves of these specimens, after yielding, differed from that of the S6M4 control specimen, and showed a slight increase in the load after yielding. The S6M4-10 specimen, which had no sound region in the side member and a partly decayed main member, showed a low load at the initial deformation. The S6M4-8 specimen, which had no sound region in the boundary between the main and side members, showed a lower load, and had an uncertain yield point on the load-slip curve.

The S8M2-2 specimen, which had a sound side member and a partly decayed main member, exhibited almost the same load-slip curve as the S8M2 control specimen. The S8M2-5 specimen, which had a sound side member and the main member with no sound region, showed a low load at the initial deformation. The S8M2-8 specimen, which had no sound region in the boundary between the main and side members, showed a smaller load than the S8M2-5 specimen. The S8M2-10 specimen, which had no sound region in either of the main and side members, showed an even lower load than the S8M2-8 specimen. The nailed connections of the S6M4 and S8M2 group also showed a low load during the initial deformation, when the main and side members had a decayed region in the boundary between them, and the decrease of the sound region in the main and side members caused the decrease in the load after yielding.

\section{Yield load}

Slip of sound nailed connections subjected to a lateral force is increased in proportion to the load, and then the slip is largely increased regardless of a slight increase in the load. Yield could be defined as status that the inclination of load-slip curves is significantly changed. Figure 9 shows the envelope load-slip curves of sound nailed connections and the inclination of tangent of load-slip curves $(\mathrm{d} P / \mathrm{d} \delta)$. Regardless of the specification of nailed connections, the value of $\mathrm{d} P / \mathrm{d} \delta$ showed a sharp decrease up to $1 \mathrm{~mm}$ of slip and a slight decrease after $2 \mathrm{~mm}$ of slip. This result could be interpreted to mean that the yield load will be in the range of 1-2 mm of slip. The authors searched for the method to determine yield load. The yield load of nailed connections with three specifications could be obtained from the method as follows: The straight line, fit to the initial linear portion of load-slip curve, is shifted in the positive $x$-direction by one-third of the nail diameter, and yield load is defined as the intersection of this line and the load-slip curve. The initial straight line was determined by the line that goes through the origin and the point on the curve corresponding to slip of one-tenth of the nail diameter. In this study, the yield load was determined using this method based on the concept described above, although the yield load of 


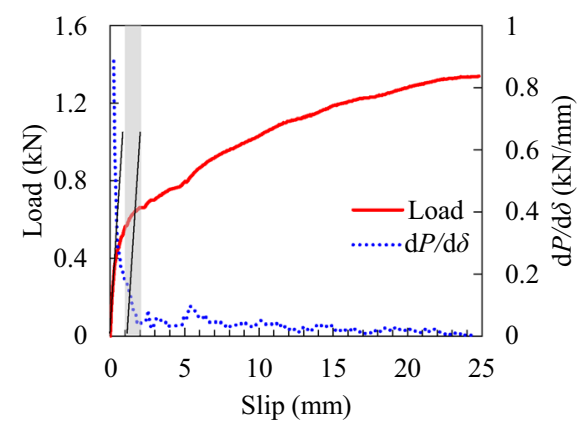

(a) S3M7 specimens

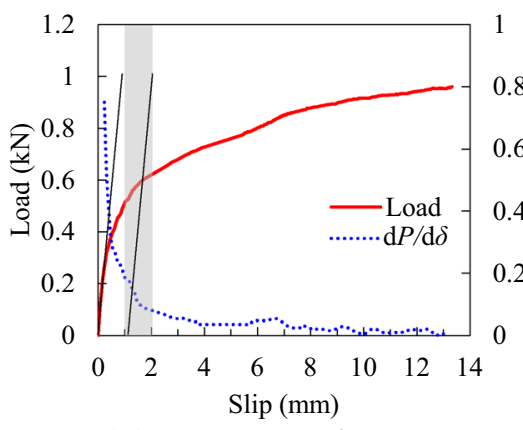

(b) S6M4 specimens

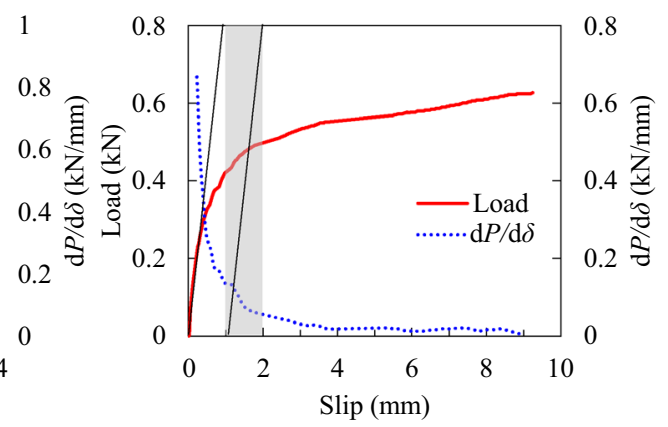

(c) S8M2 specimens

Fig. 9 Inclination of tangent of load-slip curves

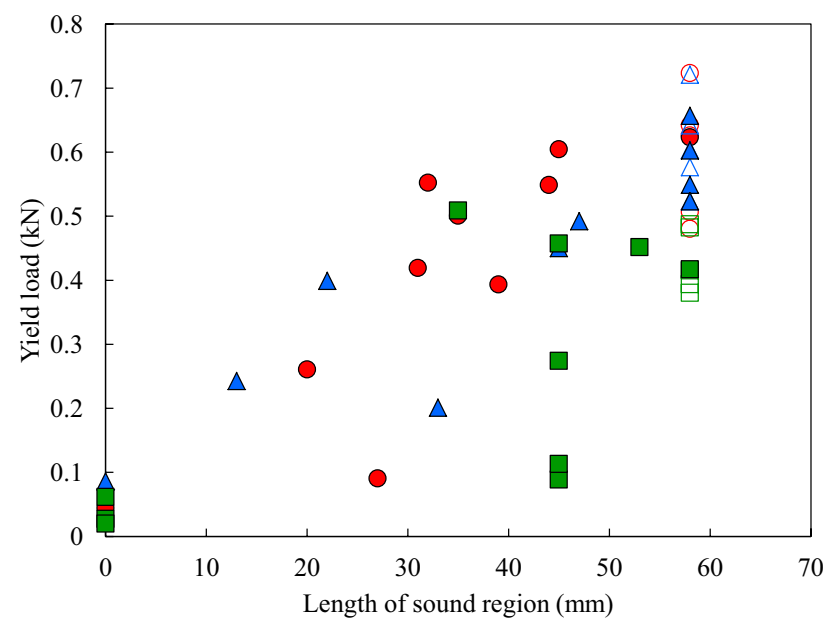

Fig. 10 Relationship between yield load and sound distance in boundary between main and side members. Open circles: control specimens of S3M7; filled circles: decay-treated specimens of S3M7; open triangles: control specimens of S6M4; filled triangles: decaytreated specimens of S6M4; open squares: control specimens of S8M2; filled squares: decay-treated specimens of S8M2

connections made with mechanical fastener are often determined according to EN12512 [30], and ASTM D-5652 [32]. When the slip corresponding to yield load was calculated from the load-slip curves as shown in Fig. 9, that obtained from the method in this study was $1.67-1.72 \mathrm{~mm}$. The slip corresponding to yield load obtained according to EN12512 and ASTM D-5652 was 0.60-0.94 and 0.55-0.59 mm, respectively. The slip corresponding to yield load evaluated according to EN12512 and ASTM D-5652 was in the range which showed a sharp decrease of the value of $\mathrm{d} P /$ $\mathrm{d} \delta$. To compare the values obtained from same evaluation method, this method also applied to the calculation of yield load of decay-treated specimens.

When the nailed connections had no sound region in the boundary between the main and side members, they showed a low load at both the initial and later deformations (Fig. 8).

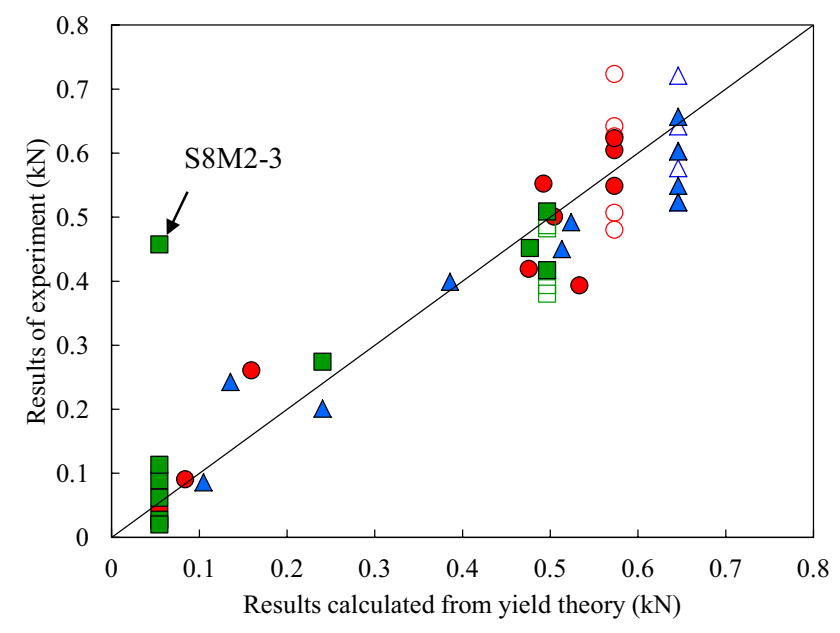

Fig. 11 Comparison of results of the experiment with results calculated based on the yield theory. Symbols: see Fig. 10

Therefore, the yield load was compared with the length of sound region in the boundary between the main and side members and the comparison was shown in Fig. 10. The specimens that had no sound region in the main and side members showed significantly lower yield loads. When the specimens had the length of sound region in a range of $13-53 \mathrm{~mm}$, they tended to show a smaller yield load as the length of sound region was decreased. However, they showed a large variable of yield load. It is considered that the yield load of nailed connections with part decay will be affected by not only the length of sound region in the boundary between the main and side members, but also the mechanism of nailed connections subjected to lateral force.

\section{Comparison to experiment}

The calculation of yield load based on yield theory was conducted on the nailed connections with part decay. The embedding strength of sound $\left(18.3 \mathrm{~N} / \mathrm{mm}^{2}\right)$ and decay 
$\left(1.26 \mathrm{~N} / \mathrm{mm}^{2}\right)$ regions were obtained from the embedding tests. The distance $\left(t_{i j}\right)$ used in calculation was based on the values shown in Table 4 . The yield moment $\left(M_{\mathrm{y}}\right)$ of the nails was the plastic section modulus $\left(d^{3} / 6\right)$ times the yield bending strength $\left(599 \mathrm{~N} / \mathrm{mm}^{2}\right)$ described above.

Figure 11 shows the relations between yield load obtained in the experiments and those calculated from the yield theory. The yield load of S8M2-3 specimens obtained in the experiments was larger than that calculated from the yield theory. The S8M2-3 specimen had the decay in neighborhood of nailing point and a little part of the region from nailing point was sound. Because the model in this study established requirements that the embedding strength is not changed along parallel to the grain in the decay region, the calculated values might be underestimated. However, the calculated yield load of the other specimens was close to those obtained in the experiments. These results indicate that the equation, which is derived in this study based on the yield theory, is useful for estimating the yield load of the nailed connection with partial and whole decay.

\section{Conclusions}

Loading tests were conducted on nailed connections that were exposed to a brown rot fungus, $F$. palustris; the connections differed according to three specifications based on combinations of different side member thicknesses and nail lengths in the main member. The yield load of nailed connections with part decay was calculated based on yield theory. The results obtained can be summarized as follows:

1. The relations between load and slip of nailed connections were significantly affected by the decay. The nailed connections with decay showed a low load during the initial deformation when the main and side members had a decayed region in the boundary between them. The decrease of the sound region in the main member caused the decrease in the load after yielding.

2. The yield load of the nailed connections decreased as the length of sound region in the boundary between main and side members was decreased; however, there showed large variable of yield load.

3. The model, which had sound and decay regions within a member, was constructed based on yield theory. The yield load of nailed connection as calculated based on the yield theory agreed with the results obtained experimentally when significant decay in the direction parallel to the grain was produced in members. This result indicates that the yield theory can estimate the yield load of nailed connections not only with a sound member, but also with a partly and wholly decayed member.
Acknowledgements The authors wish to thank Ms. Nozomi Saito, Hokkaido University, for her assistance in the decay treatment. This work was supported by JSPS KAKENHI Grant numbers 26850107 and $17 \mathrm{~K} 07870$.

\section{References}

1. Wilcox W (1978) Review of literature on the effects of early stages of decay on wood strength. Wood Fiber 9:252-257

2. Mizumoto S (1966) The effect of decay caused by Gloeophyllum trabeum on the strength properties of Japanese red pine sap-wood. J Jpn For Soc 48:7-11

3. Toole E (1971) Reduction in crushing strength and weight associated with decay by rot fungi. Wood Sci 3:172-178

4. Brown F (1963) A tensile strength test for comparative evaluation of wood preservatives. For Prod J 13:405-412

5. Kennedy R (1958) Strength retention in wood decayed to small weight losses. For Prod J 8:308-314

6. Curling S, Clausen C, Winandy J (2002) Relationships between mechanical properties, weight loss, and chemical composition of wood during incipient brown-rot decay. For Prod J 52:34-39

7. Takanashi R, Ishihara W, Sawata K, Sano Y, Azuma T, Mori M, Koizumi A, Sasaki Y, Hirai T (2014) Fractography of shear failure surface of softwood decayed by brown-rot fungus. J Wood Sci 60:186-193

8. Sawata K, Sasaki T, Doi S, Iijima Y (2008) Effect of decay on shear performance of dowel-type timber joints. J Wood Sci $54: 356-361$

9. Kent S, Leichti R, Rosowsky D, Morrell J (2004) Effects of wood decay by Postia placenta on the lateral capacity of nailed oriented strand board sheathing and Douglas fir framing members. Wood Fiber Sci 36:560-572

10. Kent S, Leichti R, Rosowsky D, Morrell J (2005) Effects of decay on the cyclic properties of nailed connections. J Mater Civil Eng 17:579-585

11. Toda M, Mori M, Ohashi Y, Hirai T (2010) Effects of wood decay on the shear performance of nailed timber joint (in Japanese). Mokuzai Gakkaishi 56: 41-47

12. Mori T, Tanaka K, Nakahata T, Kawano K, Yanase Y, Kurisaki H (2014) Estimation of shear strength of nail driven into decayed wood. In: World conference on timber engineering, Quebec City, Canada

13. Toda M, Mori M, Takahashi H, Karimata T, Hirai T (2013) Effect of decay in structural wooden panels on the shear performance of nailed timber joints (in Japanese). Mokuzai Gakkaishi 59:152-161

14. Sawata K, Honda K, Hirai T, Koizumi A, Sasaki Y (2010) Effect of member thickness and nail length projecting from main member on shear performance of single shear nailed joints (in Japanese). Mokuzai Gakkaishi 56:317-325

15. Sawata K, Takiuchi H, Toda M, Sasaki T, Mori M (2008) Effect of wood decay on embedding performance of wood and shear performance of dowel-type joints and nailed joints. In: World conference on timber engineering, Miyazaki, Japan

16. Takanashi R, Sawata K, Sasaki Y, Koizumi A (2017) Withdrawal strength of nailed joints with decay degradation of wood and nail corrosion. J Wood Sci 63:192-198

17. Johansen K (1949) Theory of timber connections. Int Assoc Bridge Struct Eng (IABSE) Pub 9:249-262

18. EN 1995-1-1 (2004) Eurocode 5-design of timber structuresgeneral common rules and rules for buildings. European Committee for Standardization (CEN), Brussels 
19. AWC (2015) National design specification for wood construction with commentary. American Wood Council, Leesburg

20. Bejtka I, Blass H (2002) Joints with inclined screws. In: 35th Meeting of CIB-W18. Kyoto, Japan

21. Johnsson H, Lukaszewska E, Stehn L (2004) Nailed timber joints with a thick interlayer. In: World conference on timber engineering, Lahti, Finland

22. Sawata K, Sasaki T, Kanetaka S (2006) Estimation of shear strength of dowel-type timber connections with multiple slottedin steel plates by European yield theory. J Wood Sci 52:496-502

23. Uibel T, Blass H (2006) Load carrying capacity of joints with dowel type fasteners in solid wood panels. In: 39th Meeting of CIB-W18, Florence, Italy

24. Standard for structural design of timber structures (2006) Architectural Institute of Japan, Tokyo, pp 266-278

25. Sawata K, Kawamura H, Takanashi R, Ohashi Y, Sasaki Y (2016) Effects of arrangement of steel plates on strength of dowel-type cross laminated timber joints with two slotted-in steel plates subjected to lateral force. In: World conference on timber engineering, Vienna, Austria
26. Ishiyama H, Koshihara M (2009) Experimental study on the performance of the nailed joint with the rust (in Japanese). J Struct Constr Eng 74:2281-2289

27. JIS A 5508 (2009) Nails (in Japanese). Japanese Industrial Standard, Tokyo

28. JIS G 3532 (2011) Low carbon steel wires (in Japanese). Japanese Industrial Standard, Tokyo

29. ISO 10984-1 (2009) Timber structures-dowel-type fastenerspart 1: determination of yield moment. ISO, Geneva

30. EN12512 (2002) Timber structures - test methods-cyclic testing of joints made with mechanical fasteners. European Committee for Standardization (CEN), Brussels

31. Sawata K, Shibusawa T, Ohashi K, Castellanos J, Hatano Y (2008) Effects of density profile of MDF on stiffness and strength of nailed joints. J Wood Sci 54:45-53

32. ASTM D-5652 (1995) Standard test methods for bolted connections in wood and wood-based products. American Society for Testing and Materials, West Conshohocken 